Physics, Chemistry, and Dynamics of Interplanetary Dust

ASP Conference Series, Vol. 104, 1996

Bo A. S. Gustafson and Martha S. Hanner (eds.)

\title{
Optical Search for High Meteors in Hyperbolic Orbits
}

\author{
S.C. Woodworth \& R.L. Hawkes \\ Physics Engineering and Geoscience Dept., Mount Allison University, \\ Sackville, N.B., Canada EOA 3 CO
}

\begin{abstract}
Dual-station image intensified television studies have indicated very few meteors at heights greater than $120 \mathrm{~km}$, and few statistically significant hyperbolic orbits. However, the optimum intersection height for these studies was about $95 \mathrm{~km}$, and the relatively small fields of view resulted in a bias against high (and therefore fast) meteors. We have developed height sensitivity correction factors, and found that short baseline television studies resulted in relatively little bias against high meteors, and the absence of meteors above $120 \mathrm{~km}$ appears to be real. We report preliminary results from a three-station, image-intensified video meteor detection system sensitive to apparent magnitude about $+9.5 \mathrm{with}$ optimum intersection heights $115-125 \mathrm{~km}$. We have detected neither particularly high meteors nor meteors in clearly hyperbolic orbits. We conciude that the proportion of true hyperbolic meteors in the mass range $10^{-4}$ to $10^{-6} \mathrm{~kg}$ is less than a few percent, and that optical meteors corresponding to meteoroids in this mass range do not ablate at heights above approximately $120 \mathrm{~km}$. We suggest several ways to reconcile these results with southern hemisphere radar studies.
\end{abstract}

\section{Introduction}

In this paper we will consider two related, but distinct, questions: Are there significant numbers of faint optical (image intensified television) meteors at great heights (i.e., 120 to $140 \mathrm{~km}$ above the Earth's surface)? Do some of these faint optical meteors correspond to heliocentric hyperbolic orbits? While the two questions are related (hyperbolic meteors have higher geocentric velocities and would be expected to ablate at greater heights), they are distinct and separate. For example, one could envision other mechanisms for high meteors which do not involve hyperbolic velocities (e.g. meteoroids with lower boiling point).

The question of interstellar meteors has been shrouded in controversy for decades. Öpik (1941), based largely on an analysis of visual observations conducted using the rocking mirror method claimed that as many as $70 \%$ of the meteors were in hyperbolic orbits, some at several times the parabolic velocity limit. Stohl (1970) reviewed photographic results, finding that the percentage of meteors in hyperbolic orbits range from about 2 to $24 \%$ in 8 different studies. However, when the data is divided into categories according to the precision of the orbital determinations, only 0 to $2 \%$ of the most precise orbits are hyperbolic. This should not necessarily be interpreted as implying that all observed hyperbolic meteors are due to measurement inaccuracies, but rather as confining the percentage of photographic hyperbolic meteors to at most $1 \%$. More recently Hajdukova (1994) has analysed the photographic meteor orbits in the IAU Meteor Data Center, reaching the conclusion that not more than $0.2 \%$ of photographic meteors are of interstellar origin. We have reviewed results from two station image intensified video networks, and find that 6-11\% of television meteors have apparent heliocentric hyperbolic orbits, although the less precise nature (compared to photographic) of these orbital reductions leaves open the question of hyperbolic television meteors. 
Recently the Ulysses dust experiment has given compelling evidence for detection of interstellar dust (Grün et al., 1994, Baguhl et al., 1995) from a direction consistent with the flow of gas in the solar neighbourhood. The near or total absence of hyperbolic orbit photographic meteors and the presence of interstellar dust detected by spacecraft is not in conflict. The smallest masses observed in the photographic studies are of the order of $10^{-5} \mathrm{~kg}$ while the largest masses detected by the Ulysses dust detectors are of the order of $10^{-12} \mathrm{~kg}$.

Baggaley et al. (1993a) announced the detection, using the New Zealand AMOR radar (Baggaley et al., 1993b) of significant numbers (0.9\%) of meteors in hyperbolic orbits. The minimum masses of meteors detected by this radar are of the order of $10^{-9} \mathrm{~kg}$ (about +13 magnitude).

As mentioned at the outset, one can have meteors ablate at great heights by mechanisms other than implied hyperbolic velocities, while one can also have mildly hyperbolic velocities without unduly great ablation heights. Nevertheless, in this paper we link the work of Steel (1988), which suggests, on the basis of HF meteor radar results, that many meteoroids ablate at heights greater than originally assumed (right up to $140 \mathrm{~km}$ ). It would be encouraging to confirm the presence of these high meteors through optical detection. Image intensified video methods (Hawkes and Jones, 1986; Hawkes, 1993) are capable of extending the minimum meteoroid mass observed by almost a factor of 100 , to masses of $10^{-7} \mathrm{~kg}$.

\section{Correction of Television Meteor Height Distributions}

If we exclude those studies which concentrated on shower meteors, there have been three major published multistation video meteor studies (Hawkes et al., 1984; Sarma and Jones, 1985; Ueda and Fujiwara, 1994). An analysis of the heights detected in these studies indicate very few meteors above $120 \mathrm{~km}$. However, it can be argued that there was a significant bias against very high and low meteors, since the optimum intersection height was set to approximately $95 \mathrm{~km}$ and the relatively small fields of view (typically $13^{\circ}$ ) result in significant bias against high (and low) meteors. To our knowledge, this bias has not been quantitatively studied previously. The finite meteor trail length is comparable to the field of view, and therefore correction for the bias is not simply a matter of geometric observing area. We simulated, using the observing geometry of each study, 40,000 meteors at each height over an area which encompassed all possible regions seen by any camera in the system. We generated meteors with midpoints at heights of $137.5 \mathrm{~km}$ to $67.5 \mathrm{~km}$, at intervals of $5 \mathrm{~km}$, and assumed a random "radiant" distribution except that we excluded meteors with zenith angles greater than $80^{\circ}$. Based on the observational trail length results for television meteors of this magnitude by Jones and Hawkes (1975), we used a vertical trail length of $7 \mathrm{~km}$ for all simulated meteors. Each meteor trail was divided into six points, and using the actual observing geometry we determined whether each of those points were visible from station 1 , station 2 or both. In order to obtain triangulation data for a meteor trail using the method of intersection of planes (Hawkes et al., 1993) it is only necessary to have any segment of the trail viewed from both stations (e.g. the early part of the trail from one station, and the last portion from the other, with no common segment, is sufficient). We therefore defined a meteor as "in common" if at least one of our 6 points was visible from each station (but not necessarily the same point). In order to have a beginning height there is the additional requirement that at least one station see the first point on the trail.

To correct an observed height distribution, we multiplied the raw number of meteors at each height by the number from the simulation at maximum overlap and divide by the simulated number at this particular height. Because the collecting area (for any individual station) increases with height, in some cases the actual "optimum overlap" is slightly higher than the intersection height of the midpoint of the screens. We also applied a correction for the greater range of high meteors, assuming an inverse square 
loss of intensity and a log normal mass distribution with a mass distribution index of 2.0.

In Figure 1 we show the raw and corrected rates for one portion ( $377 \mathrm{~km}$ baseline) of the Sarma and Jones (1985) data. It can be seen that while the number of high meteors is undersampled, there is still clear evidence that there are very few optical meteors above $125 \mathrm{~km}$. This is confirmed by the simulations for the other short baseline $(<40 \mathrm{~km})$ geometries (the height sample of the longer baseline, $102.6 \mathrm{~km}$, Hawkes et al., 1984 data is strongly biased).

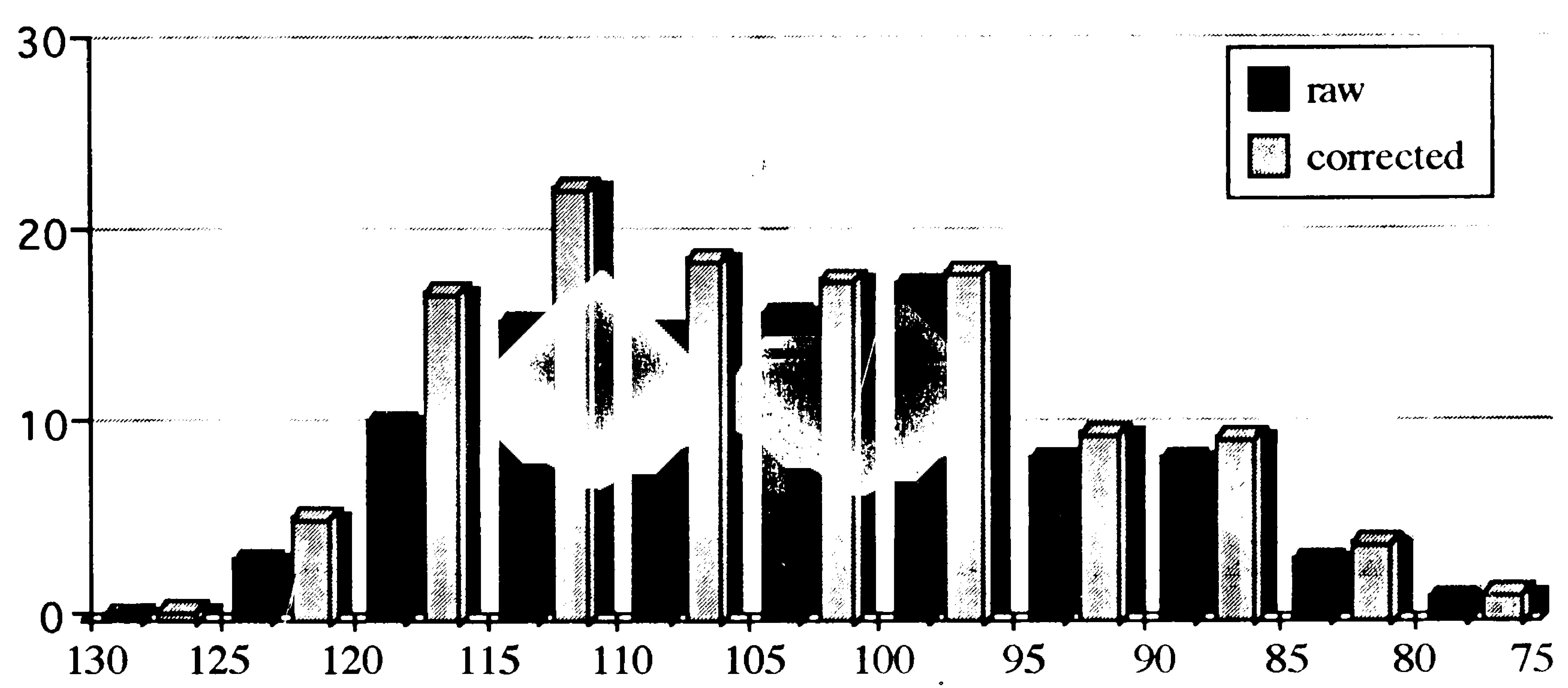

Figure 1 Plot of percentage of meteors (raw and after height sensitivity corrections) as a function of height interval (beginning heights) for image intensified video data from the Sarma and Jones (1985) study

\section{Observational Study}

Starting in June 1995 we began an observational study aimed at detecting high optical meteors and those in hyperbolic orbits. From our location the best part of the year to detect interstellar meteors, if concentrated near the galactic centre as the Ulysses results suggest (Baguhl et al., 1995), or if concentrated in the direction of the apex of the sun's motion, is the April-July period. We set the intersection height to the range $115-125 \mathrm{~km}$ to strongly bias the sample in favour of high, fast meteors and employed three stations with optimum geometry to reduce uncertainties. The parameters of the observing stations are given below

\begin{tabular}{|c|c|c|c|c|c|c|}
\hline station & longitude & latitude & camera & field of view & lens & sensit \\
\hline Alma, NB & $64^{\circ} 55^{\prime} 35^{\prime \prime} \mathrm{W}$ & $45^{\circ} 36^{\prime} 00^{\prime \prime} \mathrm{N}$ & $M C P-C C D$ & $13.4^{\circ} \times 10.5^{\circ}$ & $50 / f 0.95$ & 9.5 \\
\hline Sackville, NE & $64^{\circ} 22^{\prime} 22^{\prime \prime} W$ & $45^{\circ} 53^{\prime} 33^{\prime \prime} \mathrm{N}$ & $M C P-C C D$ & $4.9^{\circ} \operatorname{circ}$ & $300 / f 4$ & 10 \\
\hline Wallace, NS & $63^{\circ} 28^{\prime} 12^{\prime \prime} \mathrm{W}$ & $45^{\circ} 48^{\prime} 14^{\prime \prime} \mathrm{N}$ & MCP-SIT & $24.9^{\circ} \times 18.4^{\circ}$ & $50 / f 1.2$ & 8.5 \\
\hline
\end{tabular}

Table 1 Observational Parameters

The baseline between the Alma and the Wallace sites was $115.7 \mathrm{~km}$, between Alma and Sackville $53.9 \mathrm{~km}$, and between Sackville and Wallace, $70.6 \mathrm{~km}$. We operated the cameras for six nights near new moon in June, 1995. Simulations indicate that we had relatively good sensitivity through the $110-140 \mathrm{~km}$ height range, and that coverage dropped off sharply below $95 \mathrm{~km}$. We therefore expected to detect few normal height meteors, but our system should detect extremely high meteors with good efficiency

Analysis is complete for the first two nights (approx. 10.t hr observing time per station). The average number of meteors detected per station was about 65 , although 
only $8 \%$ were in common. The highest meteor detected was $115 \mathrm{~km}$, and none have clearly hyperbolic orbits. Analysis is continuing on three more nights of data from June, 1995.

\section{Discussion}

Considering the strong bias in favour of high meteors (tempered with the small sample size) we conclude that at most a few percent of meteors of this size are hyperbolic, and that there is no evidence for meteor luminosity above about $120 \mathrm{~km}$. These results are partially in conflict with sensitive southern hemisphere radio observations. One possible explanation is that some constituent of the meteoroid (perhaps the "glue" in the two component dustball meteoroid model of Hawkes \& Jones, 1975) ablates at very great heights, producing ionization but not luminosity. A second possibility is that the ionizing efficiency increases with velocity far more rapidly than the luminous efficiency factor, hence biasing radar results in favour of very fast meteors. A third possibility is that there is a flux of very fast, high meteors only at masses smaller than the limiting mass (approximately $5 \times 10^{-8} \mathrm{~kg}$ ) of the television observations. Finally, it is possible that most fast, high hyperbolic meteors come from a radiant which strongly favours southern hemisphere observers.

\section{Acknowledgements}

This work was supported by a Natural Sciences and Engineering Research Council of Canada Research Grant. One of the authors (SW) acknowledges support from a Mount Allison University Summer Research Fellowship. We thank James Flynn who operated the Wallace camera and Rob Eaton for suggestions and assistance.

\section{References}

Baggaley, W.J., Taylor, A.D., \& Steel, D.I., 1993a, in Meteoroids \& Their Parent Bodies, J. Stohl \& I.P Williams, Bratislava: Astron. Inst. Slovak Acad. Sci., 53

Baggaley. W.J., Taylor, A.D., \& Steel, D.I., 1993b, in Meteoroids \& Their Parent Bodies. J. Stohl \& I.P. Williams, Bratislava: Astron. Inst. Slovak Acad. Sci., 245

Baguhl, M., Hamilton, D.P., Grün, E., Dermott, S.F., Fechtig, H., Hanner, M.S., Kissel, J., Lindblad, B.-A., Linkert, D., Linkert, G., Mann, I., McDonnell, J.A.M., Morfill, G.E., Polanskey, C., Riemann, R., Schwehm, G., Staubach, P., \& Zook, H.A., 1995, Science, 268, 1016

Grün, E., Gustafson, B., Mann, I., Morfill, G.E., Staubach, P., Taylor, A., \& Zook, H.A., 1994, Astron. Astrophys., 286, 915

Hajdukova, M., 1994, Astron. Astrophys., 288, 330

Hawkes, R.L., 1993, in Meteoroids \& Their Parent Bodies, J. Stohl \& I.P. Williams,

Bratislava: Astron. Inst. Slovak Acad. Sci., 227

Hawkes, R.L. \& Jones, J., 1975, Mon. Not. R. astr. Soc., 173, 339

Hawkes, R.L. \& Jones, J., 1986, Q. Jl. R. astr. Soc., 27, 569

Hawkes, R.L., Jones, J., \& Ceplecha, Z., 1984, Bull. Astron. Inst. Czech., 35, 46

Hawkes, R.L., Mason, K.I., Fleming, D.E.B., \& Stultz, C.T., 1993, in Proceedings

International Meteor Conference, Belgium: IMO, 28

Jones, J. \& Hawkes, R.L., 1975, Mon. Not. R. astr Soc., 171, 159

Jones, J. \& Sarma, T., 1985, Bull. Astron. Inst. Czech., 36, 103

Öpik, E.J., 1941, Pub. Astron. Observ. Tartu, 30, 6

Sarma, T.\& Jones, J., 1985, Bull. Astron. Inst. Czech., 36, 9

Steel, D.I., 1988, in Dust in the Universe, M.E. Bailey \& D.A. Williams, Cambridge:

Cambridge Univ Press, 187

Stohl, J., 1970, Bull. Astron. Inst. Czech., 21, 10

Ueda., M. \& Fujiwara, Y., 1994, Earth, Moon \& Planets (in press) 